\title{
BMJ Open Influence of metabolic profiles on the safety of drug therapy in routine care in Germany: protocol of the cohort study EMPAR
}

\author{
Tatjana Huebner (D) , ${ }^{1}$ Michael Steffens, ${ }^{1}$ Roland Linder, ${ }^{2}$ Jochen Fracowiak, ${ }^{1}$ \\ Daria Langner, ${ }^{2}$ Marco Garling, ${ }^{2}$ Felix Falkenberg, ${ }^{2}$ Christoph Roethlein, ${ }^{3}$ \\ Willy Gomm, ${ }^{3}$ Britta Haenisch, ${ }^{1,3,4}$ Julia Stingl ${ }^{5}$
}

To cite: Huebner T, Steffens M, Linder R, et al. Influence of metabolic profiles on the safety of drug therapy in routine care in Germany: protocol of the cohort study EMPAR. BMJ Open 2020;10:e032624. doi:10.1136/ bmjopen-2019-032624

- Prepublication history and additional material for this paper are available online. To view these files, please visit the journal online (http://dx.doi org/10.1136/bmjopen-2019032624).

Received 27 June 2019 Revised 05 December 2019 Accepted 07 January 2020

Check for updates

(C) Author(s) (or their employer(s)) 2020. Re-use permitted under CC BY-NC. No commercial re-use. See rights and permissions. Published by BMJ.

For numbered affiliations see end of article.

Correspondence to Professor Julia Stingl; jsting|@ukaachen.de

\section{ABSTRACT}

Introduction Pre-emptive testing of

pharmacogenetically relevant single-nucleotide polymorphisms can be an effective tool in the prevention of adverse drug reactions and therapy resistance. However, most of the tests are not used as standard in routine care in Germany because of lacking evidence for the clinical and economical benefit and their impact on the usage of healthcare services. We address this issue by investigating the influence of pharmacogenetic profiles on the use of healthcare services over an extended period of several years using routine care data from a statutory health insurance company. The goal is to provide clinical evidence whether pre-emptive pharmacogenetic testing of metabolic profiles in routine care in Germany is beneficial and cost-effective.

Methods and analysis The EMPAR

(Einfluss metabolischer Profile auf die

Arzneimitteltherapiesicherheit in der Routineversorgung) study is a non-interventional cohort study conducted to analyse pharmacogenetic risk factors that are important for drug therapy by means of endpoints relevant for healthcare. The analysis is based on pharmacogenetic profiles and statutory health insurance data. We perform pharmacogenetic, pharmacoepidemiological and pharmacoeconomic analyses using health care utilisation scores and machine learning techniques. Therefore, we aim to include about 10000 patients ( $\geq 18$ years) insured by the health insurance provider Techniker Krankenkasse. The study focuses on patients with prescriptions of anticoagulants and prescriptions of cholesterol-lowering drugs. Also, a screening for special pharmacogenetic characteristics will be performed in patients with at least one Y57.9! diagnosis (Complication of medical and surgical care: drug or medicament, unspecified). Outcomes include the utilisation of health insurance services, the incidence of incapacity for work and costs for drugs and treatment.

Ethics and dissemination The protocol was approved by the Ethics Committee of the Medical Faculty, University of Bonn (Lfd. Nr. 339/17). The results of this research project will be published in scientific open access journals and at conferences.

Trial registration number German Clinical Trials Register, DRKS00013909.
Strengths and limitations of this study

EMPAR is the first study in Germany to analyse pharmacogenetic data matched with statutory health insurance data to evaluate drug safety in routine care.

- The Techniker Krankenkasse routine healthcare database provides reliable information to analyse the influence of pharmacogenetic profiles on the utilisation of healthcare services.

- Possible difficulties in distinguishing between causal diagnoses and adverse drug reactions are addressed by additional information from treatment-related questionnaires provided by study participants.

\section{INTRODUCTION}

Adverse drug reactions (ADRs) and therapy resistances increase morbidity and mortality of patients and thus are a clinical problem in routine care. They also complicate drug therapy and exert an economic burden on the healthcare system due to resulting follow-up costs. ${ }^{1-4}$ Up to $6.5 \%$ of hospitalisations in Germany are assumed to be a consequence of ADRs. ${ }^{56}$ ADRs and therapy resistances can be induced by extrinsic causes such as drug interactions or medication errors that are avoidable, but also by the individual response to drugs which is influenced by pharmacogenetic variability. ${ }^{7-12}$ Patients can be ultra-rapid, extensive, intermediate or poor metabolisers for a certain drug dependent on the involved pharmacogenetic variants. Therefore, due to individual differences in drug metabolism, the same dosage can lead to different drug concentrations, efficacy and safety of therapy. ${ }^{13-16}$

In the last years, data on the influence of pharmacogenetic differences with a high degree of evidence from clinical studies and systematic meta-analyses could be used for therapeutic recommendations and 
guidelines on pharmacogenetic tests. ${ }^{17-22}$ Such guidelines are provided and constantly updated by the "Clinical Pharmacogenetics Implementation Consortium' (CPIC) and the Dutch Pharmacogenetics Working Group (DPWG) established by the Royal Dutch Association for the Advancement of Pharmacy. These guidelines and recommendations are based on the pharmacogenetics expertise from research and clinical practice. ${ }^{23-25}$

Correspondingly, pharmacogenetic testing options constantly improve in accuracy and due to discovery of novel variants. They are available for a wide range of genes associated with severe drug-gene interactions and became affordable over time. ${ }^{26-28}$ Also, the development of artificial intelligence techniques to support the clinical interpretation of the complex genetic data is progressing. It can be highly useful for a future application of pharmacogenetic testing in daily practice. ${ }^{29-32}$

Although pharmacogenetic testing is a promising und evolving tool in precision medicine, pre-emptive testing, except for mandatory diagnostics for certain prescriptions, is not covered by insurance companies and not adequately used as standard of care in Germany in most cases. ${ }^{33}$ Several studies indicate that pharmacogenetics can promote the reduction of healthcare costs by preventing ADRs and can increase patient's safety in therapy with drugs. ${ }^{34-38}$ To promote coverage of pharmacogenetic testing by healthcare financiers, more pharmacoepidemiological and pharmacoeconomic studies on the benefit of pharmacogenetic tests are warranted. ${ }^{39}$ These studies could encourage the clinical utilisation of pharmacogenetic testing, the expansion of health insurance coverage to this field and the implementation of relevant trainings for a professional application of pharmacogenetics in daily care. ${ }^{4041}$ An expert report commissioned by the German Bundestag outlined the potential of pharmacogenetics in routine care. ${ }^{42}$ Therefore, for future directions in this field, the EMPAR (Influence of Metabolic Profiles on the Safety of Drug Therapy in Routine Care) study will analyse whether the use of pharmacogenetic testing could reduce healthcare expenditures and provide benefits for patients, medical practitioners and health insurance providers in Germany.

\section{METHODS AND ANALYSIS \\ Trial design}

The EMPAR study is a non-interventional cohort study, which is conducted to analyse the impact of metabolic profiles based on pharmacogenetic testing on drug safety in routine care. Therefore, pharmacogenetic profiles of participants are investigated. The genetic variant information provided by these profiles is matched with statutory health insurance data. Pharmacoepidemiological and pharmacoeconomic analyses are conducted for endpoints such as usage of healthcare services and healthcare costs.

The study includes three different groups that are defined by the initial prescription of certain drugs and the relevant International Classification of Diseases (ICD)-10 diagnoses. Due to corporate policies, data of insurants can only be provided from 2013 onwards. For each participant group, 1 year without prescription is considered as baseline for analysis. Therefore, the initial prescription is defined as a prescription event of the drug of interest after at least 1 year without a recorded prescription of the drug. Insurants are surveyed via a questionnaire on their initial prescription of the drug of interest to gain additional, supportive information on this issue.

The first group includes patients with initial prescription of anticoagulants such as clopidogrel, clopidogrel plus acetylsalicylic acid, prasugrel, ticagrelor, ticlopidine, phenprocoumon, acenocoumarol, warfarin, dabigatran, apixaban, rivaroxaban and edoxaban in 2014 and 2015 and with or without at least one ADR associated with bleeding or a thromboembolic event after initial prescription. The second group includes patients with initial prescription of cholesterol-lowering drugs such as simvastatin, lovastatin, pravastatin, atorvastatin, fluvastatin, cerivastatin, rosuvastatin and pitavastatin in 2014 and 2015 and with or without at least one ADR associated with muscle pain after initial prescription. In the anticoagulant and cholesterol-lowering drug groups, the pharmacogenetic profiles of patients with ADRs and without ADRs after initial prescription are compared. To identify new single-nucleotide polymorphisms (SNPs) and SNP combinations involved in ADRs, additionally, a screening for special metabolic profiles is performed in a third subgroup that consists of persons with at least one ICD (International Classification of Diseases and Health Related Disorders)-10 Y57.9! diagnosis in 20142017. Prior to the selection of this third participant group, an ICD-10 code screening for suitable diagnoses was performed. Also, the Techniker Krankenkasse (TK) database was screened by TK research associates to ensure that a sufficient amount of potential participants can be recruited for this group. The Y57.9! diagnosis includes complications of medical and surgical care due to drugs or medicaments that were not specified. Thereby, the drugs potentially causing adverse effects in therapeutic use were correctly selected and properly administered in therapeutic or prophylactic dosage.

The aim is to recruit about 10000 insurants of the health insurance provider TK who are at least 18 years of age. In the course of this study, we analyse the patients' relevant metabolic risk profiles concerning side effects or resistance to therapy to identify potential improvement of drug safety and modification of healthcare costs. The long-term goal is to determine the feasibility of the implementation of pre-emptive pharmacogenetic tests in routine care for an optimised drug therapy and treatment.

\section{Study setting}

EMPAR is a cooperation project of the Federal Institute for Drugs and Medical Devices (BfArM), the German Centre for Neurodegenerative Diseases (DZNE) and the statutory health insurance provider TK. It is based on data of TK insurants in Germany. 


\section{Recruitment procedures}

TK insurants who meet the inclusion criteria and do not have any of the specified exclusion criteria receive study information and an informed consent form from their health insurance provider TK. Participants receive buccal swap material from a trust centre after they have provided informed consent. Participants are enrolled in the study in case they send in their buccal swab and successfully provide a high-quality DNA sample for determination of their pharmacogenetic profile.

\section{Participants}

Inclusion criteria

1. TK insurant.

2. Aged 18 years or older.

3. Initial prescription of anticoagulants or cholesterollowering remedy in 2014-2015 or at least one Y57.9! diagnosis in 2014-2017.

4. Written informed consent.

5. Successfully provided pharmacogenetic profile.

\section{Exclusion criteria}

1. Inapplicable metabolic profile results.

2. Oncological phenotype (All ICD-10 C-diagnoses and D0x, D4x, D37, D38, D39).

3. Severe F-diagnoses (ICD-10: F0x.x, F2x.x, F7x.x, F8x.x, F31.x, F33.x, F38.x, F39.x, F42.x, F43.x, F44.x, F60.x, F61.x, F62.x, F63.x, F69.x, F91.x, F92.x, F93.x, F94.x, F95.x, F98.x, F1x.2, F1x.3, F1x.4, F1x.5, F1x.6, F1x.7, F1x.8, F1x.9, F30.1, F30.2, F30.8, F30.9, F32.2, F32.3, F32.8, F32.9, F34.8, F34.9, F45.2, F45.4, F45.8, F45.9, F48.1, F48.8, F48.9, F50.4, F50.5, F53.1, F53.8, F53.9, F65.2, F65.3, F65.4, F65.6, F65.8, F65.9, F68.1, F68.8, F90.1).

4. Known genetic hematopoietic diseases on initial prescription of anticoagulant (ICD-10 code: D55, D56 D57，D58，D61.0，D64.0，D64.4，D66，D67，D68.0, D68.1, D68.2, D71, D72.0, D74.0, D80.0, D82).

5. Y69! diagnosis (unspecified incident during surgical and medical care) in parallel with Y57.9! diagnosis.

6. Myopathy, myositis or muscle pain before initial prescription of cholesterol-lowering drugs.

7. TK customer management criteria.

\section{Trial outcomes}

On the basis of routine care data provided by the health insurance provider TK, the study will examine whether differences in pharmacogenetic profiles have an impact on the incidence of medication problems and therefore, the utilisation of statutory health insurance services. Furthermore, we analyse whether there is a modification of costs for health insurance services based on individual metabolic profiles. Several current studies provide results on cost differences of drug therapy with and without support by pharmacogenetic testing in the therapeutic decision. Thereby, the evaluations on cost reduction vary significantly across drugs and conditions. ${ }^{374344}$ With regard to the drug-gene combinations evaluated in our study, supportive evidence of ADR risk reduction and of cost effectiveness is available for clopidogrel-CYP2C19 testing; still information is scarce for other drug-gene combinations and economic evaluations of pharmacogenetics (PGx) panel testing are underrepresented. ${ }^{37} 43 \mathrm{~A}$ randomised control study in the USA which evaluated the impact of pharmacogenetic testing service on healthcare costs, suggested a possible annual saving of US\$621 per patient in the population analysed. ${ }^{45}$ With our study we want to add additional evidence based on lower-cost PGx multigene panel testing and routine healthcare records of a German population.

\section{Primary outcome}

The health insurance provider TK provides routine care data. Derived from these data, primary outcomes are incidence of incapacity for work and utilisation of health insurance services for example, hospitalisation due to ADRs, referral to a specialist due to medication problems, change of medication during the observation period. With these outcomes, we assess the impact of metabolic profiles on the incidence of ADRs and resistance to therapy.

\section{Secondary outcomes}

Secondary outcomes include the drug costs, treatment and sickness benefit. The aim is to identify a possible modification of costs for health insurance services by preemptive testing in routine care. Thus, we examine the effect of pharmacogenetic profiles on the incidence of ADRs and costs due to health insurance services.

\section{Data collection}

Data protection is executed according to the TMF (Technology and methods platform for networked medical research) series 'Guideline for Data Protection in Medical Research Projects'. ${ }^{46}$ The service laboratory represents the biobank module, the TK the clinical module and the BfArM the research module. The trust centre is responsible for identity management and pseudonymisation service.

A pseudonym protects each dataset. The insurant pseudonym is generated by the TK and is linked to healthcare data. A trust centre assigns a genotype pseudonym (G-pseudonym) and a secondary data pseudonym (S-pseudonym) to the insurant pseudonym and creates an assignment table for merging of the different datasets. Participants who provided informed consent receive a questionnaire and a buccal swab test kit by the trust centre that records their address information with the insurant pseudonym provided with the written informed consent. The questionnaire is adjusted for each group and contains questions regarding the initial prescription of the drugs of interest and associated ADRs (online supplementary file). Also, questions on the general perception of drugs on the basis of the Beliefs about Medicine Questionnaire by Horne $e t a t^{47}$ are included, supplemented by one question on pharmacogenetics and three questions on 
herbal medicines for an evaluation of medical beliefs. ${ }^{47}$ Participants send the completed questionnaire and the buccal swab samples marked only with the G-pseudonym to a specialised service laboratory for DNA-extraction and sample registration (DKMS Life Science Lab). Samples are prepared according to genotyping requirements and send to Agena Bioscience for genotype determination. The list of genotypes is forwarded for quality control and derivation of the metabolic profiles to researchers at the BfArM. A list of G-pseudonyms linked to quality controlled and usable metabolic profiles is forwarded to the trust centre where it is translated into an S-pseudonym/insurant pseudonym list. The health insurance provider TK receives this S-pseudonym/insurant pseudonym list, identifies participants by the insurant pseudonym, and anonymises and forwards the respective healthcare data only with the associated S-pseudonym to the BfArM. This way, the TK never has access to the genotype-associated pseudonym, while BfArM researches do not receive the participant data-associated pseudonym. BfArM researchers merge the anonymised datasets via the S-pseudonym and G-pseudonym assignment table that is provided by the trust centre. The merged data are analysed by researchers of the DZNE, BfArM and the TK (figure 1). Before anonymisation of data, the remaining DNA samples are stored by the service laboratory that performed the DNA-extraction. After anonymisation, the samples are stored in a biobank of the BfArM in Germany. They will be discarded after 15 years.

\section{Patient and public involvement statement}

The EMPAR study involved no patient and public contribution beyond the study participation described in this article.

\section{Data management}

Anonymised datasets will be stored in a secure electronic database at the BfArM, where also the data management and quality assessment of the study database will be hosted. Dataset extractions will be generated for pharmacogenetic, pharmacoepidemiological and pharmacoeconomic

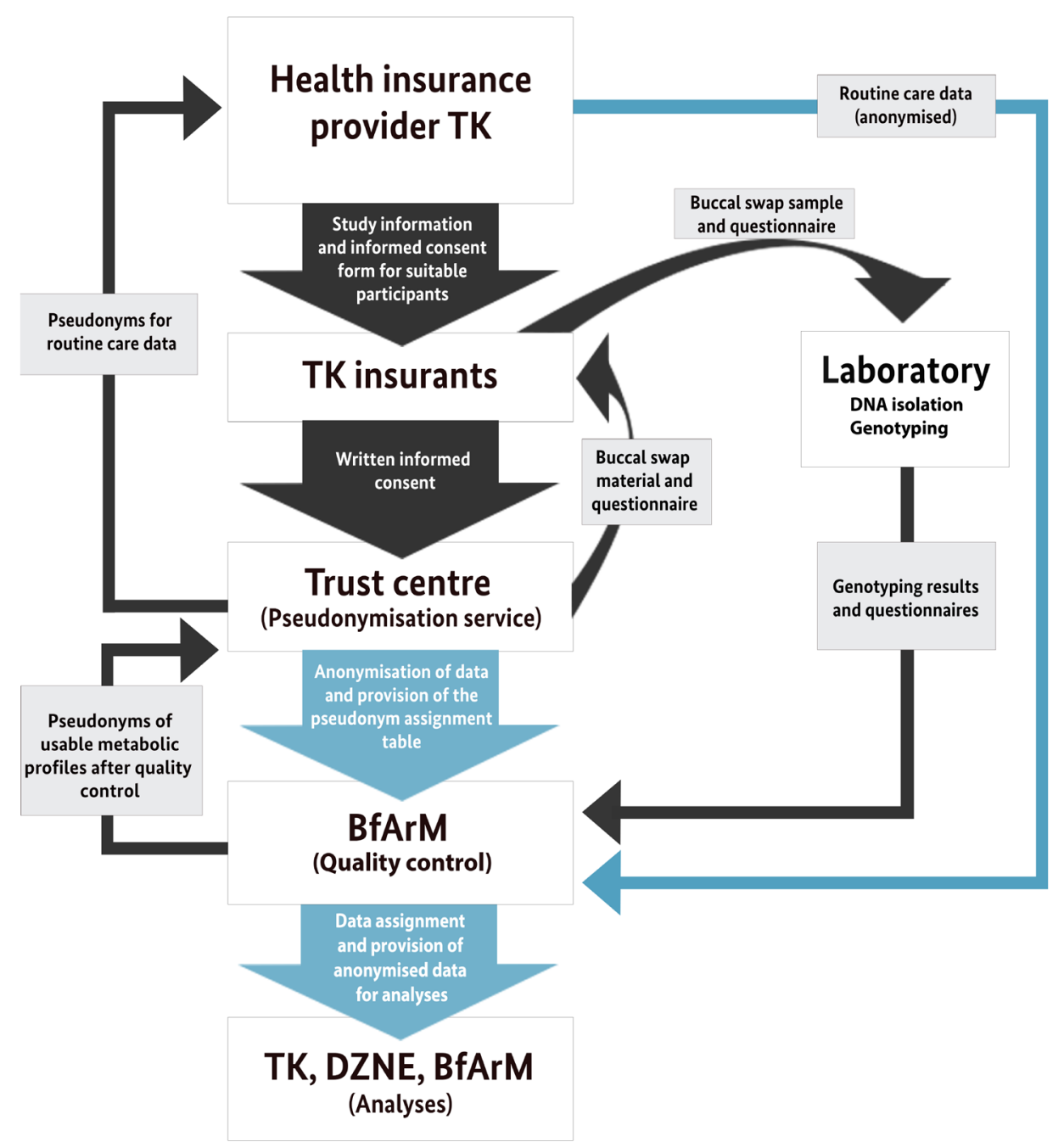

Figure 1 Data management. BfArM, Federal Institute for Drugs and Medical Devices; DZNE, German Centre for Neurodegenerative Diseases, TK, Techniker Krankenkasse. 
analyses and analysed in common by researches of the BfArM, the DZNE and the TK.

\section{Statistical analysis}

We examine the effect of metabolic profiles on drug safety in routine care. Therefore, we compare participant groups with initial prescription of the drugs of interest in 2014 and 2015 and at least one of the investigated ADRs after initial prescription with a control group. The control group comprises participants with initial prescription of the drugs of interest in 2014 and 2015 without the investigated ADR after initial prescription. Investigated ADRs in the anticoagulant group are ICD-10 diagnoses associated with thromboembolic or bleeding events. Those in the cholesterol-lowering drug group encompass diagnoses associated with muscle pain and myopathy. Additionally, a screening for special metabolic profiles of insured persons with at least one Y57.9! diagnosis (ICD10 ) is performed during the observation period. For this screening, no additional control group is recruited. For statistical analysis, data on the pharmacogenetic profile will be matched with routine care records and the questionnaire results via the according pseudonyms.

Statistical design and analysis are performed by researchers of BfArM, DZNE and TK

\section{Pharmacogenetic analysis}

For pharmacogenetic analysis, quality of genetic primary data will be stringently controlled. Possible genotyping mistakes will be identified on gene level by classical indicators such as marker and person call rates, HardyWeinberg equilibrium, allelic frequencies and haplotype frequencies. Data will be compared with present databases such as the Single Nucleotide Polymorphism Database (dbSNP), the Database of Genomic Structural Variation (dbVar) and the Clinically Relevant Sequence Variations (ClinVar) archive . Haplotype-IDs (haplotype identifiers according to star-allele nomenclature) of the super alleles will be determined by the allele status of single markers of the pharmacogenes of interest with help of the CPIC haplotype set translation tables. On the basis of the CPIC guidelines and the Human Cytochrome P450 Allele Nomenclature Database, the individual haplotypes, repectively star-alleles, can be used to derive the metabolic phenotype of a participant for the relevant pharmacogenes.

\section{Pharmacoepidemiological analysis}

For pharmacoepidemiological analysis, we use matched primary data (genetic data) and secondary data (healthcare data), provided by TK. We examine parameters that correlate with drug exposure, therapy resistance and ADRs. Those parameters include for example individual dosing of drugs, utilisation of health insurance services, burden of disease and prescription of medication. Further parameters are economic outcomes such as costs for drugs, treatment and sickness benefit. For statistical evaluation, multivariate regression analysis is used taking into account also propensity and genetic sum scores. Thus, we investigate whether genetic polymorphisms that determine the metabolic profile in drug therapy impact the above-mentioned patient-relevant endpoints. Established comorbidity and healthcare utilisation scores such as Charlson, Elixhauser, ICD-10 structural model classification, Rx Risk and Chronic Disease Score are integrated into the statistical model to address potential confounders such as multimorbidity and polypharmacy. We further plan to include potentially relevant drug-drug interactions for suitable subgroups (eg, based on metabolic profile) into our analysis.

\section{Pharmacoeconomic analysis}

Parameters for pharmacoeconomic analyses are costs for drugs and treatments taking into account the costs for pharmacogenetic testing. For estimation of diseasespecific total costs, costs for outpatient care and inpatient treatment are calculated and the total costs of the test and the control group are compared in a matched pairs analysis. Multivariate regression analysis, propensity score methods and artificial neuronal network approaches are applied.

\section{DISCUSSION}

Between 1995 and 2014, the summary of product characteristics of about $15 \%$ of all centrally approved drugs contained pharmacogenetic information. Also, the beneficial potential of pharmacogenetics in preventive healthcare was confirmed in previous and current studies. ${ }^{19} 354849$ However, despite of the growing evidence and importance of pharmacogenetic assessment, ${ }^{27} 3750-53$ the implementation of pharmacogenetic testing in standard routine care is not achieved in Germany yet. Already in 2005, an expert report on behalf of the German Bundestag regarding the status and perspectives of pharmacogenetics was published. The report provided a series of tasks for Health Technology Assessment (HTA) which focused on the utility of pharmacogenetic tests in clinical practice and, thus, already considered pharmacogenetics on the future agenda. On the basis of European studies, the report pointed out that HTA can contribute to evaluating the benefits of pharmacogenetic testing for patients, to presenting its possible consequences for the health system, and to identifying options for action. ${ }^{42} 5455$

However, studies on the effects of pharmacogenetic profiles on the utilisation of healthcare services in crosssector care are lacking in Germany. Therefore, the EMPAR study uses routine data of a German statutory health insurance provider and patient-derived pharmacogenetic profiles to close this gap. It investigates the potential of pharmacogenetic testing to be implemented as pre-emptive testing in drug therapy in routine care. Thereby, it addresses the important clinical need to identify approaches that meet the current and future developments and use contemporary possibilities for a beneficial and cost-effective advancement in healthcare. This study 
is the first study in Germany to analyse the pharmacogenetic data matched with statutory health insurance data for an evaluation of drug safety in routine care. The results of this study on the effect of pharmacogenetic profiles on drug safety will provide insights into the clinical utility of pharmacogenetic testing in clinical practice in Germany. EMPAR represents a milestone in HTA for future directions in the field of pharmacogenetics in German routine care.

\section{Ethics and dissemination}

The protocol was approved by the Ethics Committee of the Medical Faculty, University of Bonn (Lfd. Nr. $339 / 17)$. The study started in January 2018. Recruitment was initiated in July 2018 and is expected to be finalised by the end of the year 2019. Written informed consent is obtained from all study participants. The results of this research project will be published in scientific open access journals and at conferences.

\section{Author affiliations}

${ }^{1}$ Research Division, Federal Institute for Drugs and Medical Devices, Bonn, North Rhine-Westphalia, Germany

${ }^{2}$ Techniker Krankenkasse, Hamburg, Germany

${ }^{3}$ Population Health Sciences, German Centre for Neurodegenerative Diseases, Bonn, North Rhine-Westphalia, Germany

${ }^{4}$ Centre for Translational Medicine, University of Bonn, Bonn, North Rhine-

Westphalia, Germany

${ }^{5}$ Institute for Clinical Pharmacology, RWTH Aachen University, Aachen, North RhineWestphalia, Germany

Acknowledgements The authors thank Kathrin Worm, Jeanette Hoffmann and Arne Brettschneider for the administrative support at short notice.

Contributors MS, RL, BH and JS jointly conceived the study, developed the study design and received funding for the study. TH was the study coordinator. JF contributed to ID and database management. DL, MG and FF coordinated the selection of insurants and managed the mailing of cover letters from the Techniker Krankenkasse. MS, CR and WG designed the statistical analyses. TH wrote the first draft of the manuscript. All authors critically revised the manuscript and approved the final version. Due to equal contribution TH and MS share the first authorship and $\mathrm{BH}$ and JS share the last authorship.

Funding This work was supported by the Innovation Fund of the Federal Joint Committee (grant number: 01VSF16047). The funding body reviewed and approved the design of the study. The funder is not involved in data collection, analysis, and interpretation and in writing the manuscript.

Competing interests None declared.

Patient consent for publication Not required.

Provenance and peer review Not commissioned; externally peer reviewed.

Open access This is an open access article distributed in accordance with the Creative Commons Attribution Non Commercial (CC BY-NC 4.0) license, which permits others to distribute, remix, adapt, build upon this work non-commercially, and license their derivative works on different terms, provided the original work is properly cited, appropriate credit is given, any changes made indicated, and the use is non-commercial. See: http://creativecommons.org/licenses/by-nc/4.0/.

ORCID iD

Tatjana Huebner http://orcid.org/0000-0003-4548-1234

\section{REFERENCES}

1 Formica D, Sultana J, Cutroneo PM, et al. The economic burden of preventable adverse drug reactions: a systematic review of observational studies. Expert Opin Drug Saf 2018;17:681-95.
2 Lee JW, Aminkeng F, Bhavsar AP, et al. The emerging era of pharmacogenomics: current successes, future potential, and challenges. Clin Genet 2014;86:21-8.

3 Sultana J, Cutroneo P, Trifirò G. Clinical and economic burden of adverse drug reactions. J Pharmacol Pharmacother 2013;4:73-7.

4 Batel Marques F, Penedones A, Mendes D, et al. A systematic review of observational studies evaluating costs of adverse drug reactions. Clinicoecon Outcomes Res 2016;8:413-26.

5 Rottenkolber D, Schmiedl S, Rottenkolber M, et al. Adverse drug reactions in Germany: direct costs of internal medicine hospitalizations. Pharmacoepidemiol Drug Saf 2011;20:626-34.

6 Schurig AM, Böhme M, Just KS, et al. Adverse drug reactions (ADR) and emergencies. Dtsch Arztebl Int 2018;115:251-258-8.

7 Al-Jumaili AA, Doucette WR. Comprehensive literature review of factors influencing medication safety in nursing homes: using a systems model. J Am Med Dir Assoc 2017;18:470-88.

8 Bahar MA, Setiawan D, Hak E, et al. Pharmacogenetics of drugdrug interaction and drug-drug-gene interaction: a systematic review on CYP2C9, CYP2C19 and CYP2D6. Pharmacogenomics 2017;18:701-39.

9 Miklosz J, Kalaska B, Mogielnicki A. Pharmacogenetic considerations of anticoagulant medication. J Physiol Pharmacol 2018;69.

10 Reynolds KK, Pierce DL, Weitendorf F, et al. Avoidable drug-gene conflicts and polypharmacy interactions in patients participating in a personalized medicine program. Per Med 2017;14:221-33.

11 Schmiedl S, Rottenkolber M, Szymanski J, et al. Preventable ADRs leading to hospitalization - results of a long-term prospective safety study with 6,427 ADR cases focusing on elderly patients. Expert Opin Drug Saf 2018;17:125-37.

12 Turner RM, Park BK, Pirmohamed M. Parsing interindividual drug variability: an emerging role for systems pharmacology. Wiley Interdiscip Rev Syst Biol Med 2015;7:221-41.

13 Blancas I, Rodriguez Gonzalez CJ, Muñoz-Serrano AJ, et al. Influence of CYP2D6 polymorphism in the outcome of breast cancer patients undergoing tamoxifen adjuvant treatment. Journal of Clinical Oncology 2018;36:e12521-21.

14 Brown S-A, Pereira N. Pharmacogenomic impact of CYP2C19 variation on clopidogrel therapy in precision cardiovascular medicine. J Pers Med 2018;8.

15 Gaedigk A, Sangkuhl K, Whirl-Carrillo M, et al. Prediction of CYP2D6 phenotype from genotype across world populations. Genet Med 2017;19:69-76.

$16 \mathrm{Wu} \mathrm{AH}$. Drug metabolizing enzyme activities versus genetic variances for drug of clinical pharmacogenomic relevance. Clin Proteomics 2011;8:12.

17 Early Breast Cancer Trialists' Collaborative Group (EBCTCG). Aromatase inhibitors versus tamoxifen in early breast cancer: patientlevel meta-analysis of the randomised trials. Lancet 2015;386:13411352-52.

18 Goetz MP, Sangkuhl K, Guchelaar H-J, et al. Clinical pharmacogenetics implementation Consortium (CPIC) guideline for CYP2D6 and tamoxifen therapy. Clin Pharmacol Ther 2018;103:770-7.

19 Mega JL, Walker JR, Ruff CT, et al. Genetics and the clinical response to warfarin and edoxaban: findings from the randomised, double-blind engage AF-TIMI 48 trial. Lancet 2015;385:2280-7.

20 Johnson JA, Caudle KE, Gong L, et al. Clinical pharmacogenetics implementation Consortium (CPIC) guideline for PharmacogeneticsGuided warfarin dosing: 2017 update. Clin Pharmacol Ther 2017;102:397-404.

21 Hicks JK, Sangkuhl K, Swen JJ, et al. Clinical pharmacogenetics implementation Consortium guideline (CPIC) for CYP2D6 and CYP2C19 genotypes and dosing of tricyclic antidepressants: 2016 update. Clin Pharmacol Ther 2017;102:37-44.

22 Moriyama B, Obeng AO, Barbarino J, et al. Clinical Pharmacogenetics Implementation Consortium (CPIC) Guidelines for CYP2C19 and Voriconazole Therapy. Clin Pharmacol Ther 2017;102:45-51.

23 Bank PCD, Caudle KE, Swen JJ, et al. Comparison of the guidelines of the clinical pharmacogenetics implementation Consortium and the Dutch pharmacogenetics Working group. Clin Pharmacol Ther 2018;103:599-618.

24 Swen JJ, Nijenhuis M, de Boer A, et al. Pharmacogenetics: from bench to byte--an update of guidelines. Clin Pharmacol Ther 2011;89:662-73.

25 Caudle KE, Klein TE, Hoffman JM, et al. Incorporation of pharmacogenomics into routine clinical practice: the clinical pharmacogenetics implementation Consortium (CPIC) Guideline development process. Curr Drug Metab 2014;15:209-17. 
26 Dawes M, Aloise MN, Ang JS, et al. Introducing pharmacogenetic testing with clinical decision support into primary care: a feasibility study. CMAJ Open 2016;4:E528-34.

27 van Schaik RH. Ifcc Task force on pharmacogenetics: Prof Dr. Maurizio Ferrari PDMNPDMPPDH-JGPDRvS. clinical application of pharmacogenetics: where are we now? Ejifcc 2013;24:105-12.

28 Weitzel KW, Cavallari LH, Lesko LJ. Preemptive panelbased pharmacogenetic testing: the time is now. Pharm Res 2017;34:1551-5.

29 Ginsburg GS, Phillips KA. Precision medicine: from science to value. Health Aff 2018;37:694-701.

30 Grossi E, Podda GM, Pugliano M, et al. Prediction of optimal warfarin maintenance dose using advanced artificial neural networks. Pharmacogenomics 2014;15:29-37.

31 Clifton DA, Niehaus KE, Charlton $P$, et al. Health informatics via machine learning for the clinical management of patients. Yearb Med Inform 2015;10:38-43-43.

32 Tang J, Liu R, Zhang Y-L, et al. Application of Machine-Learning models to predict tacrolimus stable dose in renal transplant recipients. Sci Rep 2017;7:42192.

33 Wurcel V, Perche O, Lesteven D, et al. The value of companion diagnostics: overcoming access barriers to transform personalised health care into an affordable reality in Europe. Public Health Genomics 2016;19:137-43.

$34 \mathrm{Ji}$ Y, Skierka JM, Blommel JH, et al. Preemptive pharmacogenomic testing for precision medicine: a comprehensive analysis of five actionable pharmacogenomic genes using next-generation DNA sequencing and a customized CYP2D6 genotyping cascade. J Mol Diagn 2016;18:438-445-45.

35 Pink J, Pirmohamed M, Lane S, et al. Cost-Effectiveness of pharmacogenetics-guided warfarin therapy vs. alternative anticoagulation in atrial fibrillation. Clin Pharmacol Ther 2014;95:199-207.

36 Plöthner M, Ribbentrop D, Hartman J-P, et al. Cost-Effectiveness of pharmacogenomic and pharmacogenetic Test-Guided personalized therapies: a systematic review of the Approved active substances for personalized medicine in Germany. Adv Ther 2016;33:1461-80.

37 Verbelen M, Weale ME, Lewis CM. Cost-Effectiveness of pharmacogenetic-guided treatment: are we there yet? Pharmacogenomics J 2017;17:395-402.

38 Plumpton $\mathrm{CO}$, Roberts D, Pirmohamed M, et al. A systematic review of economic evaluations of pharmacogenetic testing for prevention of adverse drug reactions. Pharmacoeconomics 2016;34:771-93.

39 Kapoor R, Tan-Koi WC, Teo Y-Y. Role of pharmacogenetics in public health and clinical health care: a SWOT analysis. Eur J Hum Genet 2016;24:1651-7.

40 Hresko A, Haga SB. Insurance coverage policies for personalized medicine. J Pers Med 2012;2:201-16.
41 Brown JT, Gregornik D, Kennedy MJ, et al. The role of the pediatric pharmacist in precision medicine and clinical pharmacogenomics for children. J Pediatr Pharmacol Ther 2018;23:499-501.

42 TAB. Pharmakogenetik. Secondary Pharmakogenetik, 2017. Available: https://www.tab-beim-bundestag.de/de/pdf/ publikationen/berichte/TAB-Hintergrundpapier-hp013.pdf [Accessed 15 Dec 2017].

43 Zhu Y, Swanson KM, Rojas RL, et al. Systematic review of the evidence on the cost-effectiveness of pharmacogenomics-guided treatment for cardiovascular diseases. Genet Med 2020;22:475-86.

44 Krebs K, Milani L. Translating pharmacogenomics into clinica decisions: do not let the perfect be the enemy of the good. Hum Genomics 2019;13:39.

45 Saldivar J-S, Taylor D, Sugarman EA, et al. Initial assessment of the benefits of implementing pharmacogenetics into the medical management of patients in a long-term care facility. Pharmgenomics Pers Med 2016;9:1-6.

46 Dr PK, Helbing K, Ganslandt T. Leitfaden zum Datenschutz in medizinischen Forschungsprojekten, 2014.

47 Horne R, Weinman J, Hankins M. The beliefs about medicines questionnaire: the development and evaluation of a new method for assessing the cognitive representation of medication. Psychol Health 1999;14:1-24.

48 Bishop JR, Najjar F, Rubin LH, et al. Escitalopram pharmacogenetics: CYP2C19 relationships with dosing and clinical outcomes in autism spectrum disorder. Pharmacogenet Genomics 2015;25:548-54.

49 Madlensky L, Natarajan L, Tchu S, et al. Tamoxifen metabolite concentrations, CYP2D6 genotype, and breast cancer outcomes. Clin Pharmacol Ther 2011;89:718-25.

50 Berm EJJ, Looff Mde, Wilffert B, et al. Economic evaluations of pharmacogenetic and pharmacogenomic screening tests: a systematic review. second update of the literature. PLoS One 2016;11:e0146262

51 Deverka PA. Pharmacogenomics, evidence, and the role of payers. Public Health Genomics 2009;12:149-57.

52 Kim K, Magness JW, Nelson R, et al. Clinical utility of pharmacogenetic testing and a clinical decision support tool to enhance the identification of drug therapy problems through medication therapy management in polypharmacy patients. J Manag Care Spec Pharm 2018;24:1250-9.

53 Sugarman EA, Cullors A, Centeno J, et al. Contribution of pharmacogenetic testing to modeled medication change recommendations in a long-term care population with polypharmacy. Drugs Aging 2016;33:929-36.

54 Netherlands HCot. Pharmacogenetics - Advisory report, 2000.

55 University of Cambridge DoPHaPC. Wellcome trust my very own medicine. what must I know? information policy for pharmacogenetics, 2003. 